\title{
Fluoride imaging of atherosclerotic plaques: Moving from macro to microcalcifications?
}

\author{
Fabien Hyafil, MD, PhD, ${ }^{a}$ Warda Ferrag, $M D,{ }^{a}$ Chahinez Kefti, $M D,{ }^{a}$ and \\ Dominique Le Guludec, MD, PhD ${ }^{a}$ \\ a Department of Nuclear Medicine, Département Hospitalo-Universitaire FIRE, Centre Hospital- \\ ier Universitaire Bichat, Université Paris Diderot, Bichat University Hospital and UMR 1148 \\ INSERM, Assistance Publique - Hôpitaux de Paris, Paris, France
}

Received Jul 16, 2018; accepted Jul 16, 2018

doi: 10.1007/s12350-018-1385-6

\section{See related article, pp. 1064-1075}

\section{FNA ACCUMULATES IN CULPRIT ATHEROSCLEROTIC PLAQUES}

Sodium ${ }^{18} \mathrm{~F}$-fluoride sodium (FNa) is a radiotracer that has been approved for clinical skeletal bone imaging with positron emission tomography (PET) since 1972.

${ }^{18}$ Fluoride ions bind to bone after exchange of an $\mathrm{OH}$ ion tested for the identification of active and progressing calcifications in patients with aortic valve stenosis (AVS). ${ }^{1,2}$ Interestingly, the comparison of $18 \mathrm{~F}$-fluorodeoxyglucose (FDG) and FNa signals in aortic valves ${ }^{3}$ suggested that there might be a time sequence for each radiotracer in valves with high FDG uptake in relation to chronic inflammation at the initial stage of AVS, followed at the intermediate stage of AVS by FNa binding in link with an active calcification process, leading ultimately to highly calcified obstructive aortic valves. During the analysis of FNa-PET acquisitions in patients with AVS, Dweck et al. ${ }^{3}$ were intrigued by the presence of focal FNa uptake on PET in noncalcified regions of coronary arteries on CT in some of the patients. To understand more precisely the characteristics of atherosclerotic plaques that bind $\mathrm{FNa}$, they set up a clinical study ${ }^{4}$ that searched

Reprint requests: Dominique Le Guludec, MD, PhD, Department of Nuclear Medicine, Département Hospitalo-Universitaire FIRE, Centre Hospitalier Universitaire Bichat, Université Paris Diderot, Bichat University Hospital and UMR 1148 INSERM, Assistance Publique Hôpitaux de Paris, 46 rue Henri Huchard, 75018 Paris,

France; dominique.leguludec@bch.aphp.fr

J Nucl Cardiol 2019;26:1076-8.

$1071-3581 / \$ 34.00$

Copyright (c) 2018 American Society of Nuclear Cardiology. for FNa binding in coronary atherosclerotic plaques of 40 patients who had presented a recent acute myocardial infarction and 40 patients with stable angina and looked more in depth at the characteristics of coronary atherosclerotic plaques accumulating FNa with intravascular ultrasound-Virtual Histology (IVUS-VH) and coronary CT angiography (CCTA). In $93 \%$ of the patients who had presented recent acute myocardial infarction, high FNa signal was present in the stented culprit plaque in coronary arteries. In the same work, the authors also described in patients with recent acute ischemic stroke the binding of $\mathrm{FNa}$ in carotid atherosclerotic plaques ipsilateral to the stroke. The FNa signal was present on PET in all ruptured carotid plaques and associated with evidence of active calcification, macrophage infiltration, apoptosis, and necrosis on histology. In the article published in this issue of the Journal of Nuclear Cardiology, Hop et al. ${ }^{5}$ aimed at confirming that culprit carotid plaques bind more $\mathrm{FNa}$ than nonculprit lesions. After in vitro incubation of carotid plaques with FNa, they, however, did not find any significant difference in the FNa signal between lesions obtained from carotid plaques ipsilateral vs contralateral to the ischemic stroke. A possible explanation for this finding is that plaque vulnerability is a systemic process ${ }^{6}$ meaning that carotid lesions contralateral to the territory of the ischemic stroke often also present features of complicated plaques. Indeed, Hop et al..$^{5}$ confirmed that all nonculprit lesions that bound $\mathrm{FNa}$ had features of complicated plaques on histology. Carotid plaques of asymptomatic patients would have represented a better comparator to evaluate FNa binding in stable plaques, but are more difficult to collect as indications for carotid endarterectomy in this population are declining. The study of Hop, et al. ${ }^{5}$ confirms that FNa is binding to ruptured atherosclerotic plaques, but does not allow us rule on the interest of FNa to discriminate between highrisk and stable lesions. 


\section{DOES THE FNA SIGNAL IN ATHEROSCLEROTIC PLAQUES COME ONLY FROM MICROCALCIFICATIONS?}

The fact that $\mathrm{FNa}$ can accumulate in vascular segments without any calcification detectable on CT was the most intriguing finding. To unravel the mechanisms of FNa binding in atherosclerotic plaques, Irkle et al. ${ }^{7}$ compared the presence and location of FNa binding in atherosclerotic plaques detected on autoradiography with the presence of calcifications on micro-CT and histology. They found that FNa cannot penetrate into the deeper layers of macrocalcifications and only binds at their surface. FNa signal was found proportionally higher in microcalcifications of small volume but large surface than in macrocalcifications of large volume and small surface. In addition, microcalcifications in the vascular wall have an attenuation profile to X-rays that is too weak to be detected with CT explaining how FNa can be present in the vascular wall in absence of calcification on CT. Hop et al. ${ }^{5}$ confirmed after in vitro incubation of FNa of carotid atherosclerotic plaques, that regions with high FNa uptake on PET with no calcification on micro-CT did contain microcalcifications on histology, but also that $\mathrm{FNa}$ can bind to regions with macrocalcifications on CT. This is in accordance with the study of Derlin et al. ${ }^{8}$ who showed in a retrospective analysis of 75 patients imaged with wholebody FNa-PET-CT for oncological indications that only $12 \%$ of focal $\mathrm{FNa}$ signals detected in the aorta were located in regions without any calcification on CT, whereas only $12 \%$ of the vascular regions with calcifications on CT did bind FNa. FNa signal can thus be found in vascular regions without any detectable calcification on CT, but bind also to a small proportion of plaques that appear as calcified on CT. An important remaining question for the analysis of FNa-PET imaging in atherosclerotic plaques is whether these two patterns of FNa binding in the vascular wall have the same significance in terms of plaque vulnerability or if the analysis of the FNa signal should rather focus on the signal that is not associated with any macrocalcification detectable on $\mathrm{CT}$, which might be more specific for microcalcifications.

\section{DOES THE FNA SIGNAL IN ATHEROSCLEROTIC PLAQUES PREDICT VULNERABILITY?}

The detection of coronary calcifications has been used in different ways for risk assessment of cardiovascular events. The most validated approach consists in quantifying with a low-dose gated CT scan the total volume of macrocalcifications, which is referred as the coronary artery calcification score (CACS). The CACS reflects the extent and severity of coronary atherosclerotic burden in an individual. ${ }^{9}$ The increased risk of cardiovascular events observed in individuals with high $\mathrm{CACS}^{10}$ is mostly explained by the high prevalence of vulnerable plaques and myocardial ischemia in this population with severe and extensive coronary atherosclerosis rather than as a consequence of the presence of coronary calcifications. Interestingly, Budoff et al. ${ }^{11}$ evidenced that individuals with a fast annual increase in CACS ( $>100$ /year) have a higher risk of cardiovascular events than the one predicted only with their baseline CACS supporting the deleterious impact of actively calcifying processes in coronary plaques. Compared to CACS, coronary CT angiography (CCTA) has the advantage of allowing for the direct visualization of the composition of coronary atherosclerotic plaques. In patients with recent acute coronary syndromes, culprit and high-risk plaques are typically formed of large noncalcified hypodense regions on CCTA, whereas calcified plaques are often seen in patients with stable coronary artery disease (CAD). CCTA of coronary plaques confirmed that the presence of large calcifications in plaques is a marker of their stability, even though small « spotty » calcifications $(<1 \mathrm{~mm})$ can be found in high-risk plaques on CCTA. ${ }^{12}$ Complementary to morphological imaging, FDG-PET is currently the most widely used molecular imaging technique for the assessment of plaque biology. FDG is avidly taken up by activated hypoxic macrophages, and its uptake in vessel wall correlated closely with the density of macrophages determined histologically. ${ }^{13}$ High FDG uptake can be found in culprit coronary and carotid lesions ${ }^{14,15}$ and is associated with the increased risk of cardiovascular events at follow-up. ${ }^{16}$ Surprisingly, no significant correlation was found between the signal detected in coronary plaques with FDG and FNa. ${ }^{4}$ In a similar way to what has been postulated in aortic valves, FNa-PET imaging might capture advanced plaques, which developed after a chronic inflammatory phase, before their evolution toward plaque stabilization and the development of macrocalcifications detectable on $\mathrm{CT}^{3,17}$ Hop et al. ${ }^{5}$ confirmed that FNa binds in complicated atherosclerotic plaques, but ruptured plaques have characteristics different from vulnerable plaques such as increased permeability to radiotracers and the presence of intraplaque hemorrhage or thrombi that might play a role in the intensity of the FNa signal. The rationale that has been proposed to link the presence of an FNa signal and plaque vulnerability is that microcalcifications might intensify the shear stress of thin-capped atheroma and thus favor plaque rupture. ${ }^{17}$ The arguments in favor of this hypothesis are that small calcified nodules have been detected on histology in the superfical layers of ruptured coronary atherosclerotic 
plaques, ${ }^{18}$ which could increase the local stress in the fibrous cap in systole according to mathematical modeling of tissue stress in plaques. ${ }^{19}$ The current evidence that FNa-PET imaging allows for the detection of vulnerable plaques is, however, not as strong as for ruptured plaques. In a small cohort of 18 patients with stable CAD, Joshi et al. ${ }^{4}$ did detect an FNa signal in coronary atherosclerotic plaques of patients with stable CAD that had markers of plaques vulnerability on IVUS-VH, but only $45 \%$ of plaques with focal FNa uptake had microcalcifications on IVUS. A few studies $^{20,21}$ have followed patients after FNa-PET imaging of atherosclerotic plaques, but were not able to demonstrate so far the potential of $\mathrm{FNa}$ to predict cardiovascular events. The hypothesis that microcalcifications can weaken the cap and favor plaque rupture is attractive but will need to be proven in prospective studies which will monitor the evolution of plaques that bind FNa and patient clinical outcome. The incremental prognostic value of FNa-PET imaging over CACS and the analysis of coronary plaque morphology on CCTA will also need to be demonstrated.

\section{Disclosure}

F. Hyafil, W. Ferrag, C. Kefti and D. Le Guludec have nothing to disclose.

\section{References}

1. Hyafil F, Messika-Zeitoun D, Burg S, et al. Detection of 18fluoride sodium accumulation by positron emission tomography in calcified stenotic aortic valves. Am J Cardiol 2012;109:1194-6.

2. Dweck MR, Jones C, Joshi NV, et al. Assessment of valvular calcification and inflammation by positron emission tomography in patients with aortic stenosis. Circulation 2012;125:76-86.

3. Dweck MR, Jenkins WS, Vesey AT, et al. 18F-sodium fluoride uptake is a marker of active calcification and disease progression in patients with aortic stenosis. Circ Cardiovasc Imaging 2014;7:371-8.

4. Joshi NV, Vesey AT, Williams MC, et al. 18F-fluoride positron emission tomography for identification of ruptured and high-risk coronary atherosclerotic plaques: A prospective clinical trial. Lancet 2014;383:705-13.

5. Hop H, de Boer SA, Reijrink M, et al. (18)F-sodium fluoride positron emission tomography assessed microcalcifications in culprit and non-culprit human carotid plaques. J Nucl Cardiol 2018. https://doi.org/10.1007/s12350-018-1325-5.

6. Hyafil F, Schindler A, Sepp D, et al. High-risk plaque features can be detected in non-stenotic carotid plaques of patients with ischaemic stroke classified as cryptogenic using combined (18)FFDG PET/MR imaging. Eur $\mathrm{J}$ Nucl Med Mol Imaging 2016;43:270-9.
7. Irkle A, Vesey AT, Lewis DY, et al. Identifying active vascular microcalcification by (18)F-sodium fluoride positron emission tomography. Nat Commun 2015;6:7495.

8. Derlin T, Richter U, Bannas P, et al. Feasibility of $18 \mathrm{~F}$-sodium fluoride PET/CT for imaging of atherosclerotic plaque. J Nucl Med 2010;51:862-5.

9. Sangiorgi G, Rumberger JA, Severson A, et al. Arterial calcification and not lumen stenosis is highly correlated with atherosclerotic plaque burden in humans: A histologic study of 723 coronary artery segments using nondecalcifying methodology. J Am Coll Cardiol 1998;31:126-33.

10. Detrano R, Guerci AD, Carr JJ, et al. Coronary calcium as a predictor of coronary events in four racial or ethnic groups. N Engl J Med 2008;358:1336-45.

11. Budoff MJ, Young R, Lopez VA, et al. Progression of coronary calcium and incident coronary heart disease events: MESA (MultiEthnic Study of Atherosclerosis). J Am Coll Cardiol 2013;61:1231-9.

12. van Velzen JE, de Graaf FR, de Graaf MA, et al. Comprehensive assessment of spotty calcifications on computed tomography angiography: Comparison to plaque characteristics on intravascular ultrasound with radiofrequency backscatter analysis. J Nucl Cardiol 2011;18:893-903.

13. Tawakol A, Migrino RQ, Bashian GG, et al. In vivo $18 \mathrm{~F}$-fluorodeoxyglucose positron emission tomography imaging provides a noninvasive measure of carotid plaque inflammation in patients. $\mathrm{J}$ Am Coll Cardiol 2006;48:1818-24.

14. Rogers IS, Nasir K, Figueroa AL, et al. Feasibility of FDG imaging of the coronary arteries: Comparison between acute coronary syndrome and stable angina. JACC Cardiovasc Imaging 2010;3:388-97.

15. Rudd JH, Myers KS, Bansilal S, et al. Atherosclerosis inflammation imaging with 18F-FDG PET: Carotid, iliac, and femoral uptake reproducibility, quantification methods, and recommendations. J Nucl Med 2008;49:871-8.

16. Figueroa AL, Abdelbaky A, Truong QA, et al. Measurement of arterial activity on routine FDG PET/CT images improves prediction of risk of future $\mathrm{CV}$ events. JACC Cardiovasc Imaging 2013;6:1250-9.

17. Nakahara T, Dweck MR, Narula N, Pisapia D, Narula J, Strauss HW. Coronary artery calcification: From mechanism to molecular imaging. JACC Cardiovasc Imaging 2017;10:582-93.

18. Virmani R, Kolodgie FD, Burke AP, Farb A, Schwartz SM. Lessons from sudden coronary death: A comprehensive morphological classification scheme for atherosclerotic lesions. Arterioscler Thromb Vasc Biol 2000;20:1262-75.

19. Vengrenyuk Y, Carlier S, Xanthos S, et al. A hypothesis for vulnerable plaque rupture due to stress-induced debonding around cellular microcalcifications in thin fibrous caps. Proc Natl Acad Sci USA 2006;103:14678-83.

20. Blomberg BA, de Jong PA, Thomassen A, et al. Thoracic aorta calcification but not inflammation is associated with increased cardiovascular disease risk: Results of the CAMONA study. Eur J Nucl Med Mol Imaging 2017;44:249-58.

21. Ishiwata Y, Kaneta T, Nawata S, Hino-Shishikura A, Yoshida K, Inoue T. Quantification of temporal changes in calcium score in active atherosclerotic plaque in major vessels by (18)F-sodium fluoride PET/CT. Eur J Nucl Med Mol Imaging 2017;44:1529-37. 